Gareth D. Williams

Pietro Bembo On Etna. The Ascent of a Venetian Humanist

Nueva York, Oxford University Press, 2017, 416 p.

ISBN 978-0-19-027229-6

\title{
Raffaele Girardi
}

Università di Bari

raffaele.girardi@uniba.it

Al De Aetna, la prima, impegnativa prova in latino del giovane Pietro Bembo, che nella forma del moderno dialogo umanistico (di stampo classico) concentrava lo spirito nuovo e vivace del confronto fra sodali dell'ambiente letterario veneziano, è ora dedicato questo corposo studio (corredato da una vastissima bibliografia) per i tipi della Oxford University Press, nel quale il suo autore, Gareth D. Williams, inserisce un'accurata edizione in traduzione inglese dell'operetta bembiana, avendo come base la princeps del 1496, che Carlo Dionisotti rivide per l'edizione dell'Officina Bodoni di Verona (1969), curata da G. Mardersteig.

L'«Etna Idea», che Williams fissa come tema conduttore del suo libro, secondo il chiarimento offerto in apertura del cap. I, più che una definizione categorica, è un "principio dinamico", che nel De Aetna Bembo avrebbe sviluppato sulla base di un'ampia conoscenza delle numerose rappresentazioni dell'Etna che la tradizione letteraria greco-romana, da Pindaro fino al poema in esametri latini di anonimo, Aetna, aveva lasciato in eredità ai moderni. La complessità di quelle rappresentazioni, che l'autore compulsa spingendosi, sia pure solo per cenni, anche oltre l'epoca dell'anonimo (cap. 1.4), mostra, secondo Williams, la tendenza a differenziare per raddoppio le visioni del paesaggio vulcanico siciliano. Una tale "doubleness», che affiora decisamente, in particolare, nei testimoni della letteratura latina, in qualche modo ritorna come fattore caratterizzante nel dialogo bembiano, che in effetti si affida a diverse prospettive di rappresentazione del paesaggio simbolico e naturale percepito nel suggestivo viaggio siciliano dall'umanista Bembo. 
Pindaro è il primo testimone della tradizione letteraria greca preso in considerazione da Williams (cap. I), il quale esamina la prima delle sue odi Pitiche, composta in occasione della vittoria conseguita da Gerone I, tiranno di Siracusa, in una corsa di carri nei giochi Pitici organizzati a Delfi nel 470 a.C, dove il tiranno si era fatto proclamare signore della città di Etna, da lui stesso fondata nei pressi del vulcano e data in governatorato a suo figlio Deinomene. L'ode offre infatti un discorso sull'Etna che è da considerare a tutti gli effetti, come già sostiene H. M. Hine (2002), il primo, organico resoconto di un'eruzione vulcanica. Essa indugia sul mito della punizione comminata da Zeus al riottoso Tifeo, condannato a vivere confinato sotto l'Etna. Chiara l'analogia che il racconto pindarico suggerisce fra questo episodio e il grande mito della resistenza greca contro i barbari (p. 30).

I temi della Pitica I riecheggiano il racconto mitico già presente nella $T e$ ogonia di Esiodo, dove si dettagliavano le vicende originarie del conflitto fra Zeus e i Titani, la mostruosa genesi di Tifeo e il decisivo epilogo del conflitto, segnato dal fulmine di Zeus che annienta Tifeo facendolo finire sotto l'Etna. Elementi narrativi, questi ultimi, che Bembo fa risuonare nel De Aetna quando il dialogo è portato dal padre Bernardo sul tema del 'flusso' dei materiali lavici dell'Etna $(\$ 39)$.

Ma è sui modelli latini (capp. 1.1-1.4) che più in dettaglio Williams concentra l'analisi fenomenica della «doubleness», per cercare le matrici di un motivo tipico di tensione interna che affiora nel dialogo bembiano De Aetna: il dualismo fra il piano razionale e scientifico della materia 'naturale' costituita dal fenomeno vulcanico e quello più connesso all'invenzione, ossia all'Etna come tema mitico. Virgilio per primo sembrava recuperare in toni iperbolici e in chiave di aemulatio, secondo Williams, la rappresentazione del'Etna proposta da Pindaro, con un eccesso di enfasi sugli elementi 'mostruosi' della scena vulcanica che in Aen. III, 570-582 si presenta allo sguardo di Enea, appena approdato all'isola dei Ciclopi. E i mostruosi orrori del vulcano dovevano in realtà prefigurare un altro orrore, che è quello dell'incontro con Polifemo.

Nel De rerum natura, rileva Williams, Lucrezio personifica l'Etna, come farà del resto Virgilio, ma a partire da presupposti materialistici riconducibili ad Epicuro, dal quale è desunta una ben terrestre dottrina dell'assalto al cielo, una ratio naturale che, dice Williams, associando Empedocle ad Epicuro, finisce per definire un fronte comune contro la tradizionale credulità religiosa e una capacità nuova di razionalizzare i fenomeni della natura con una visione che Epicuro raccomandava come lungimirante e profonda, ossia capace di giudicare i fenomeni stessi con un nuovo senso delle proporzioni e dunque atta a considerare anche gli elementi terrificanti di un'eruzione vulcanica come un fenomeno minuto e irrilevante rispetto alla grandezza dell'universo.

Nel cap. I, 3, i, con l'esame di alcune Lettere di Seneca all'amico Lucilio e di alcuni passi delle senechiane Questioni naturali, Williams riprende gli spunti offerti da Seneca al suo discepolo sulla questione dell'Etna, ricordando che il 
loro vero intento era quello di ricevere dal suo giovane corrispondente elementi descrittivi certi dei fenomeni naturali siciliani, con i quali poter comprendere come la realtà fisica di quei fenomeni si conciliasse con la loro rappresentazione mitologica e, ad esempio, come potesse l'Etna mantenere intatta la sua forza di base pur subendo nel tempo cambiamenti di forma e misura (Lett. 79. 10).

Quanto ad Ovidio (cap. I, 3, ii), nei versi delle Metamorfosi che più direttamente riguardano la genesi e le qualità dell'Etna, ossia in 5, 346-56, dove è ripreso il mito della punizione divina del temerario Tifeo, costretto a vivere nel sottosuolo dell'Etna, che per lui ribolle, è da riscontrare un'enfatizzazione dell'elemento mitologico, in evidente e anti-razionale opposizione (rileva Williams) al punto di vista del lucreziano De rerum natura.

Nel l. XV delle ovidiane Metamorfosi, un'apparente difformità di visione (vv. 340-355) sembra orientare la rappresentazione dell'attività dell'Etna in una direzione più fisica e fenomenica, costruita su presupposti di matrice empedoclea e lucreziana, in accordo con l'impostazione filosofica dell'intero libro, che si apre con un lungo discorso di Pitagora sui cattivi sistemi di nutrizione animale e sui principi generali della mutabilità del cosmo, ai quali si connette anche un nuovo discorso sulle trasformazioni del 'respiro' (spirandi vias) dell'Etna: il loro presupposto generale essendo il principio della trasmigrazione delle anime. In realtà, la dicotomia fra scienza e mitologia, ossia la distanza fra il $1 . \mathrm{V}$ e il XV delle Metamorfosi, all'altezza dei due distinti discorsi sull'Etna, è molto meno accentuata di quanto non si creda, se si esaminano, come fa Willams, i passaggi del discorso di Pitagora che nella lezione di Ovidio più sembrano sensibili, anch'essi, al fascino dei fenomeni 'meravigliosi': è in essi che lo stile discorsivo delle Metamorfosi sembra recuperare una sua omogeneità. Sono insomma essi che ridimensionano decisamente la presunta dicotomia fra i due ritratti dell'Etna, quello mitico e quello scientifico.

La memoria stratificata della rappresentazione dell'Etna emergente dalla tradizione greco-romana riaffiora per intero, rielaborata, nelle pagine del dialogo bembiano De Aetna. Williams sottopone a verifica, nel cap. 2, i modi con i quali la pratica classica della topografia memoriale si riflette nel percorso conoscitivo del De Aetna, puntando a delineare i caratteri originali del rapporto istituito da Bembo, all'interno del suo reportage dall'Etna, fra movimento fisico del viaggio e attivazione della memoria classica (cap. 2.1), rapporto inteso come vissuta concretizzazione del legame fra una peculiare esperienza conoscitiva codificata dal passato classico e la sua riproposizione nel tempo del viaggio moderno.

Le esperienze del viaggio 'antiquario' consumate prima di Bembo sono compulsate da Williams partendo dall'ineludibile esemplarità dei memoranda epistolari di Petrarca, come quello che in una missiva al cardinale Giovanni Colonna rievoca le passeggiate fra le rovine della Roma antica: un esempio, prima che per Bembo, per i primi umanisti italiani avvezzi alla pratica del viaggio antiquario come Cristoforo Buodelmonti, un iniziatore dei nuovi metodi 
d'indagine umanistica applicata alle antiquitates elleniche, o come Ciriaco di Ancona, un colto viaggiatore incline all'inchiesta sulle antiche iscrizioni e sui monumenti dell'antica Grecia, che egli conduceva portando con sé lungo gli itinerari dell'Egeo, come vademecum, il Liber insularum Archipelaghi di Cristoforo Buondelmonti. Bembo lo chiama in causa nel dialogo con Bernardo, indicandolo come un punto di riferimento e una guida esperta per la sua stessa missione di studio intorno alle caratteristiche morfologiche dell'Etna. Non mancherà, del resto, nel dialogo bembiano sull'ascesa all'Etna anche un riferimento all'universale funzione di esempio svolta dal Petrarca scalatore del Mont Ventoux (cf. cap. 2.5), col quale Bembo si misura e dialoga avendo di certo in memoria la descrizione che di quella simbolica ascesa Petrarca aveva fatto in una lettera Familiare (4.1) a Dionigi di Borgo San Sepolcro. La nuova visione che nel De Aetna si profila del moderno 'scalatore', mosso dalla viva curiosità della realtà naturale, deve qualcosa anche al nuovo modo concepito da Petrarca di vivere, con spirito individuale, il piacere tutto umano della scoperta all'interno del paesaggio naturale.

Si allineano così, nella ricognizione di Williams, le figure di un'esperienza conversativa che esalta anche il luogo dell'incontro fra padre e figlio, ossia la villa Bozza, di proprietà dei Bembo, detta il Noniano (cf. cap. 2.4): è la componente essenziale di un paesaggio anch'esso legato alla memoria letteraria e allo spazio di una collocutio collegabile al modello del dialogo classico di marca ciceroniana.

Ma è nella concreta trattazione dell'attività vulcanica come fenomeno finalmente 'naturale' che il dialogo fra Bernardo e Pietro Bembo sull'Etna assume i caratteri di una svolta metodica, rivolta a riscattare i contenuti reali dell'esperienza osservativa condotta sul paesaggio vulcanico siciliano dalle vecchie ipoteche teocentriche e dalla spiegazione 'mitica' dell'attività eruttiva dell'Etna. Alle loro spalle, lungo un ricco itinerario, ripercorso velocemente da Williams nel cap. 2.6, scorrono, fra Medioevo e Rinascimento, le esperienze di tanti viaggiatori, primi cultori di avventure propriamente alpinistiche, con un culmine nelle curiosità naturali di un artista-scienziato come Leonardo da Vinci, protagonista di una spedizione alpina sul cosiddetto Monboso (probabilmente il Monte Rosa, secondo Williams, sulla scorta di numerosi altri studiosi: cf. p. 107).

Nella scrupolosa ricostruzione della formazione greca di Bembo, Williams ripercorre le tappe di un impegnativo apprendistato veneziano, che vede in campo un apprezzato maestro di greco come Costantino Lascaris, già autore di un'Epitome delle otto parti della lingua (ed. princ. Milano, 1476), approdato a Milano nel 1458 e, dopo varie soste in numerose città italiane, a Messina. Alla felice esperienza della scuola messinese di Lascaris così come all'idea di un più esauriente viaggio di conoscenza della realtà siciliana, congettura Williams, Bembo potette probabilmente approdare dietro consiglio dell'umanista Giorgio Valla (cap. 3.3), allo scopo di perfezionare una competenza linguistica e filologica già cospicua. Ma le radici di una inclinazione umanistica di nuo- 
vo conio, fortemente connotata in senso scientifico ed enciclopedico, sono da identificare nell'innovativa lezione di un maestro come Poliziano, venuto nel 1491 a Venezia per collazionare un suo esemplare a stampa delle commedie di Terenzio con un prezioso testimone posseduto dalla famiglia Bembo (un codice che sarebbe diventato il Vat. Lat. 3226). Williams dedica a Poliziano pagine impegnative, che lasciano intendere, come suggeriva Carlo Dionisotti, quanto a lui debba la stessa predisposizione di Bembo ad una missione turistico-culturale come quella siciliana del 1492 e ad un reportage di tipo naturalistico, a base di erudizione classica, come il De Aetna, che resta pur sempre una prova d'impegno in deroga agli obblighi più strettamente politici di un giovane rampollo della classe dirigente veneziana come Bembo. A questo contesto lagunare e alla più generale vicenda quattrocentesca dell'umanesimo veneziano Williams dedica un'attenta ricognizione (cap. 4), che fa emergere, accanto ad una figura centrale come quella di Ermolao Barbaro (un esempio sintomatico, il suo, anche rispetto alle scelte del giovane Bembo, di divorzio di un umanista dalla vita della repubblica), l'importante sodalizio di Bembo con Trifone e Angelo Gabriele, (grande amico, quest'ultimo, e dedicatario del De Aetna),

Alle sperimentali novità dell'impresa editoriale di Aldo Manuzio a Venezia, che nel 1496 produceva la prima stampa del De Aetna, è dedicato il cap. 5, dove un'attenta ricognizione di Williams spazia nella densa stagione che va dai primi incunaboli aldini fino all'introduzione del modello in ottavo dei $l i$ belli portatiles, applicato al Virgilio stampato nel 1501, passando per la prima opera in latino stampata dall'editore veneziano, che è appunto il bembiano $D e$ Aetna, un'edizione eseguita su un manoscritto recante correzioni dell'autore e consegnato all'editore subito dopo il ritorno di Bembo dal viaggio in Sicilia: uno straordinario esempio, quest'ultimo, di sintesi e di relazione simbolica fra contenuto e forma testuale, fra parola e carattere tipografico. Francesco Griffo da Bologna, artefice dell'elegante carattere romano usato per la stampa del $D e$ Aetna è non a caso anche il creatore dei caratteri greci impiegati un anno prima per la stampa della grammatica greca di Costantino Lascaris.

Sulle due diverse prospettive dalle quali Bernardo e Pietro Bembo percepiscono il paesaggio simbolico dell'Etna si sofferma analiticamente il cap. 6, sottolineando la distanza che separa i moderati entusiasmi e la gravitas dell'uomo di stato (Bernardo) dall'eccitato accostamento del 'letterato' (Pietro) alle suggestioni di un paesaggio - l'Etna - percepito come spazio della meraviglia, aperto all'attività dell'immaginazione. Mentre Bernardo si appaga con una spiegazione dei caratteri del fenomeno vulcanico tutta ripiegata sulla voce delle autorità classiche, Pietro invece, durante la scalata, punta a cogliere l'originale rilevanza figurale di quel paesaggio simbolico in termini dinamici, sostenuto da un personalissimo tratteggio dei suoi elementi morfologici e dei suoi colori (che continuano a distinguerlo, nella sua configurazione dura e selvaggia, dalle linee composte e curate della scena naturale del Noniano), in continuo ma non inerte, anzi contrastivo, paragone con le immagini accostabili dei classici greci 
(Omero, Esiodo, etc.). Insomma, secondo Williams, due ben distinti paesaggi etnei scorrono sotto lo sguardo dei lettori del De Aetna: il primo è quello razionale e meccanico percepito da Bernardo in pacifica convivenza con le testimonianze dei classici; l'altro è quello più carico di inappagate curiosità (le curiosità dell'accanito collezionista, tipico del Rinascimento, quale fu Bembo), di domande e di mistero che la fervida immaginazione del letterato Pietro, attraverso una più intensa interrogazione degli stessi auctores, da Esiodo a Virgilio, prova a formalizzare nei toni affabili ma insieme imperiosi di una diversa e nuova forma di conoscenza, che è, a suo modo, come ipotizza Williams, una proiezione del sé e una violazione, vale a dire una personalizzazione, della stessa natura oggettiva, di ciò che è già noto e codificato dalla tradizione del sapere classico. 